\title{
The effect of Wii Fit exergames on static balance and motor competence in obese and non-obese college women
}

\author{
Shima Davoodeh, Mahmoud Sheikh*, Davoud Houminiyan Sharifabadi, and Fazlolah Bagherzadeh \\ Faculty of Physical Education and Sport Sciences, University of Tehran, Tehran, Iran
}

Copyright: (c) 2020 S. Davoodeh et al. This is an open access article licensed under the Creative Commons Attribution License (https://creativecommons.org/licenses/by/4.0/).

\begin{abstract}
Background: Exergames have recently been employed for a variety of purposes in different clinical and non-clinical environments. Objective: This study aimed to assess the effect of a 6-week Wii Fit exergames intervention on the static balance and motor competence in obese and non-obese college women. Methods: To this end, 20 obese (body mass index $\geq 30 \mathrm{~kg} / \mathrm{m}^{2}$, age $19.10 \pm 0.64$ years) and 20 normal-weight women (body mass index 18.5-24.9 kg/m², age $19.25 \pm 0.78$ years) were recruited. Both groups were randomly divided into intervention and control groups so that the following four groups (10 women each) were created: obese control (OC), obese Wii Fit intervention (OWFI), non-obese control (NOC) and non-obese Wii Fit intervention (NOWFI). Bruininks Oseretsky Test of Motor Proficiency (BOT-2) and static balance test using the Wii Balance Board were used to measure motor competence and static balance progression during the pretest and posttest. All the groups performed their common daily physical activities, and experimental groups had additional Wii Fit intervention for 6 weeks ( 2 sessions per week). Results: Analysis of covariance results showed a significant improvement in the static balance and motor competence of both obese and non-obese Wii Fit groups compared to their control group counterparts (Static balance: NOC < NOWFI, $p=.04$, OC < OWFI, $p=.004$; Motor competence: NOC < NOWFI, $p=.007$, OC < OWFI, $p<.001)$. Besides, the improvement in the motor competence of the obese Wii Fit group was significantly greater than that of the non-obese one (NOWFI < OWFI, $p=.04$ ). Conclusions: In addition to the similar positive effect on the static balance of obese and non-obese college women, the use of Wii Fit exergames can improve the motor competence of obese college women more than non-obese ones.
\end{abstract}

Keywords: Wii Fit, Wii Balance Board, exergames, static balance, motor competence, obesity

\section{Introduction}

While health authorities around the world are encouraging people to participate in physical activities, physical inactivity increased worldwide from 2001 to 2016, showing $80 \%$ of world teenagers are inactive and at the same time physical activity is decreasing with age. In most countries, it also represents a girl's participation in much less activity than boys (McMahon et al., 2017; Ng, Ma, Ho, Ip, \& Fu, 2019). For example, Iranian women are at a particularly high risk of adopting sedentary behavior due to specific cultural barriers, such as restrictions regarding exercising in public (Taymoori, Rhodes, \& Berry, 2010). Accordingly, a

\footnotetext{
* Address for correspondence: Mahmoud Sheikh, Department of Motor Behavior, Faculty of Physical Education and Sport Sciences, University of Tehran, 15th St., North Kargar st., Tehran, Iran. E-mail: prosheikh@yahoo.com
}

study of adolescent Iranian girls found that $64 \%$ of individuals were in pre-adoption stages of physical activity and had on averaged $<30$ min of daily physical activity (Taymoori, Niknami, Berry, Ghofranipour, \& Kazemnejad, 2009). Related to this, there has been a documented increase in the prevalence of obesity among school-children in Iran, and it is significantly more prevalent in girls $(6.1 \%)$ than in boys $(3.3 \%$; Taymoori et al., 2010).

When young men/women make the transition into university life, they are confronted with different challenges (Jacobs et al., 2011). Many factors such as unhealthy nutrition, stress, lifestyle changes lead to the phenomenon of "The Freshman 15" that generally refers to overweight around 15 pounds $(6.81 \mathrm{~kg})$ or more in the first year of college (Cluskey \& Grobe, 2009). One of the important reasons for getting overweight during this difficult period is how to coordinate the sports program with other new responsibilities, 
which makes students decide to omit physical activities. Therefore, student period seems a crucial time to execute preventive or remedial programs for students' obesity (and its consequences) and to help them maintain an active lifestyle (Jacobs et al., 2011).

As it is shown, there is almost a negative correlation between high body mass index (BMI) and motor competence, which is partly explained by an increase in body fat mass (Cattuzzo et al., 2016; D'Hondt et al., 2013). As it is clear, being overweight also reduces the balance of the obese person due to the disfiguring of the body (Błaszczyk, Cieślinska-Świder, Plewa, ZahorskaMarkiewicz, \& Markiewicz, 2009), that may stop the likelihood of physical activity and pursuing an inactive lifestyle and/or even makes its further increase, and impedes the growth of motor competence and healthrelated fitness. This negative correlation may continue throughout the crucial years between adolescence and adulthood (Nunez-Gaunaurd, Moore, Roach, Miller, \& Kirk-Sanchez, 2013). There is also an opposite effect that having a high motor competence level and a healthy weight status will lead to greater activation of the individual and help further development of motor skills in different fields (Cattuzzo et al., 2016).

Insufficient balance or deficiency in other types of postural control in different societies is associated with an increased likelihood of injury or fall risk (Gioftsidou et al., 2013). Balance training is an effective intervention to improve static postural sway and dynamic balance both in athletes and non-athletes (Zech et al., 2010).

Exergames are active video games that require the gross motor function and thus provide a unique opportunity to increase and enhance physical activities in daily life, and at the same time, they are amusing and enjoyable (Staiano et al., 2017). One of the great advantages of active video games is Nintendo Wii console and Wii Balance Board. A popular software called Wii Fit runs on the Wii console that was originally offered to healthy people in order to use it for the sake of health and fitness; however, physical therapists and physicians have welcomed it in clinical environments. Nowadays, this software is being introduced by many researchers as a fun, low-cost, and safe tool for rehabilitation programs (especially balance) or motor function improvement (Tarakci, Ersoz Huseyinsinoglu, Tarakci, \& Razak Ozdincler, 2016; Tripette, Murakami, Ryan, Ohta, \& Miyachi, 2017). More recently, there have been numerous studies of the effectiveness of Wii Fit games on the physiological, motor, and psychological factors in clinical societies such as those who are suffering from Parkinson's disease, multiple sclerosis, and Down syndrome, especially in old ages (Negrini et al., 2017; Silva et al., 2017; Willaert et al., 2020; Yazgan, Tarakci, Tarakci,
Ozdincler, \& Kurtuncu, 2020). However, research on various effects of Wii Fit on the obese and even healthy young people is very scarce. A recent study on obese and overweight children confirmed the positive effect of a 24-week home-based exergaming intervention on improving BMI $Z$-score and cardiometabolic health (Staiano et al., 2018). In addition, no studies have been found to compare the effect of Wii Fit exergames on the motor or balance factors in obese and non-obese people. Therefore, this study seeks to assess the effect of a 6-week Wii Fit exergames intervention on the static balance and motor competence in two groups of obese and non-obese college women.

\section{Methods}

\section{Participants}

Using a convenient sampling procedure, 40 female students aged 18-20 years old from Dr. Shariati Vocational and Technical Girls College in Iran were selected to participate in this study. The inclusion criteria for student selection were: (a) students' major should be based on non-physical education, (b) they should not be an athlete and should not have regular physical activity, (c) they should not have any specific illness or surgical record, (d) they should have BMI equal to or larger than $30 \mathrm{~kg} / \mathrm{m}^{2}$ (obese) and BMI between 18.5 and $24.9 \mathrm{~kg} / \mathrm{m}^{2}$ (normal or non-obese). Based on the mentioned criteria, 20 obese and 20 non-obese college women participated in the study. All participants gave their informed consent for inclusion before they participated in the study. The study was conducted in accordance with the Declaration of Helsinki, and the protocol was approved by the Ethics Committee of Sports Sciences Research of Iran (IR.SSRI.REC.1398.610). In addition, all participants filled out a questionnaire with regard to their height, weight, age, athletic background, field of study, and health status.

\section{Procedures}

Each 20-person group was randomly divided into control and intervention (10-person) subgroups. These subgroups contained OC, OWFI, NOC, and NOWFI. The intervention groups played various active games on Wii Balance Board for 6 weeks and two days per week (35-minute sessions per person). Wii Fit games are presented in four groups: Balance Games (e.g., Heading, Ski Slalom, Table Tilt, Balance Bubble), Yoga (e.g., Palm Tree, Cobra, Standing Knee, King of the Dance), Muscle Workouts (e.g., Push-up \& Side Stand, Jackknife, Lunge, Arm and Leg Lift) and Aerobic exercises (e.g., Hula Hoop, Super Hula Hoop, Step Plus, Free Step). After each stage of the game, 
an individual's performance feedback (e.g., beginner or advanced) is given, or in some games, the individual's background is recorded in that game, and the game is replayed by another one in order to compare with each other. Various games were played in turn between individuals in four groups of three and two groups of four (considering the same BMI level per a player) and each person playing the game 35 minutes. The training course was conducted under the direct supervision of an experienced trainer in order to organize the intensity and type of various plays of each of the four training groups according to the progress of the players during the 12-session period. For the participants to better see the game, the video projectors were utilized in the college classroom in order to display the games. During this period, the control groups performed their common daily physical activities.

\section{Outcome measures}

All participants have been given Bruininks Oseretsky Test of Motor Proficiency (BOT-2; Bruininks \& Bruininks, 2005) and static balance test with the Wii Balance Board before and after the intervention.

The BOT-2 short test contains 14 items that is provided to evaluate the motor skills in children and young people aged between 4 and 21 years old. Internal consistency reliability coefficient has also been reported to be .87 for a short form between age 12-21. The test-retest reliability was .80 , and inter-rater reliability was .98 for the short form (Bruininks \& Bruininks, 2005; Hands, Licari, \& Piek, 2015). This test was scored according to the standard score where the motor competence with score $\geq 70$ is considered as well above average, 60-69 above average, 41-59 average, 31-40 below average, and $\leq 30$ well below average (Hands et al., 2015).

The static balance evaluation was performed using the Wii Balance Board single-leg balance test with open eyes. This test measures the center of balance and shows a stability percentage (Crocker, 2012). Participants were placed with the dominant leg in the middle of the Wii Balance Board while the other foot rested in contact with the knee of the support leg. The kicking a ball test was used to determine limb dominance. The hands were placed on the hips joint. Everyone had to keep this position for 30 seconds. During this time, the individual observed her position on the screen as a straight line moving in the center of a path. If imbalanced, the balance line would continue to the right or left with slight deviation, and if the imbalance was too severe, the equilibrium line would hit the walls and the test would stop. Each person was allowed to repeat the test three times, and the highest score was considered as the main score. Eventually, after 30 seconds, everyone's balance score was displayed in percentage.
Inter-rater reliability and intra-rater reliability of Wii Balance Board have been reported relatively high for aged 18 to 40 years (intra-class correlation coefficients: inter-rater reliability .79-.89, intra-rater reliability .70-.92). In addition, the high concurrent validity of the Wii Balance Board with the laboratory-based force platform has also been attained (intra-class correlation coefficient .73-.87; Park \& Lee, 2014).

\section{Statistical analysis}

Data were analyzed using IBM SPSS Statistics (Version 22 for Windows; IBM, Armonk, NY, USA). Before employing the analysis of covariance (ANCOVA), Levene's homogeneity test and Shapiro-Wilk normality test were conducted. The results showed the homogeneity test has not achieved statistical significance for both the balance $(p>.99)$ and motor competence $(p=.079)$ and Shapiro-Wilk test confirmed the normality of data distribution in all four groups $(p>.05)$. Therefore, ANCOVA was used to examine the effect of the Wii Balance Board intervention in which the dependent variables were the static balance and motor competence after the intervention. The covariance was pretest scores for the aforementioned variables. Partial eta-squared $\left(\eta_{\mathrm{p}}^{2}\right)$ was used as effect size (.01-.05 a small effect, .06-.14 a medium effect, and .14 or greater a large effect; Field, 2010). Pairwise comparisons between posttest scores were carried out using the Bonferroni test. $P<.05$ was considered statistically significant.

\section{Results}

\section{Baseline characteristics}

With regard to normal weight, 20 college women were randomly divided into two groups: the intervention group (age $19.1 \pm 0.87$ years, BMI $21.46 \pm 1.87 \mathrm{~kg} / \mathrm{m}^{2}$ ) and the control group (age $19.4 \pm 0.69$ years, BMI $22.29 \pm 2.07 \mathrm{~kg} / \mathrm{m}^{2}$ ). In addition, 20 college women with obesity were randomly divided into the intervention group (age $18.8 \pm 0.70$ years, BMI $34.21 \pm 2.32$ $\mathrm{kg} / \mathrm{m}^{2}$ ) and the control group (age $19.4 \pm 0.59$ years old, BMI $32.53 \pm 1.96 \mathrm{~kg} / \mathrm{m}^{2}$ ).

\section{Static balance outcome}

The ANCOVA test results showed a significant difference in the posttest of the static balance groups after deleting covariate (pretest) effect $(F(3,35)=8.217$, $\left.p<.001, \eta_{\mathrm{p}}^{2}=.413\right)$, and according to the Bonferroni test results, these differences were significant in both intervention groups compared to their control group counterparts (NOC < NOWFI, $p=.04$; OC < OWFI, $p=.004)$. However, given the test results of the Bonferroni test, the difference was not significant between 
the obese and non-obese intervention groups ( $p=.87$ ). These results are shown with descriptive statistics of participants static balance in Table 1 .

\section{Motor competence outcome}

In the study of posttest results of the motor competence and after omitting covariate (pretest), a significant difference was obtained $(F(3,35)=17.378, p<.001$, $\left.\eta_{\mathrm{p}}^{2}=.598\right)$ and pairwise comparisons also showed a significant difference between the motor competence posttest of obese and non-obese intervention groups as compared to their control group counterparts (NOC < NOWFI, $p=.007$; OC < OWFI, $p<.001$ ). In addition, there was a significant difference between the mean of posttest motor competence of the obese and non-obese intervention groups (NOWFI < OWFI, $p=.04$ ). These results are shown with descriptive statistics of participants' static balance in Table 2 .

\section{Discussion}

The study showed that 12-session participation in Wii Fit exergames could improve the static balance and motor competence of obese and non-obese college

Table 1

Descriptive-inferential statistics of participants' static balance (in percent)

\begin{tabular}{lccl}
\hline Group & $\begin{array}{c}\text { Pretest } \\
(M \pm S D)\end{array}$ & $\begin{array}{c}\text { Posttest } \\
(M \pm S D)\end{array}$ & $\begin{array}{c}\text { Between-groups } \\
\text { effects }\end{array}$ \\
\hline NOC & $77.10 \pm 17.79$ & $75.20 \pm 18.04$ & \\
NOWFI & $71.10 \pm 16.15$ & $83.20 \pm 13.53$ & $F(3,35)=8.217$ \\
OC & $60.20 \pm 18.85$ & $65.90 \pm 17.31$ & $p<.001$ \\
$\eta_{\mathrm{p}}^{2}=.413$ \\
OWFI & $64.30 \pm 13.23$ & $84.70 \pm 9.93$ & \\
\hline
\end{tabular}

Note. $\quad$ NOC $=$ Non Obese Control; NOWFI $=$ Non Obese Wii Fit Intervention; $\mathrm{OC}=$ Obese Control; OWFI $=$ Obese Wii Fit Intervention.

Table 2

Descriptive-inferential statistics of participants' motor competence

\begin{tabular}{lccl}
\hline Group & $\begin{array}{c}\text { Pretest } \\
(M \pm S D)\end{array}$ & $\begin{array}{c}\text { Posttest } \\
(M \pm S D)\end{array}$ & $\begin{array}{c}\text { Between-groups } \\
\text { effects }\end{array}$ \\
\hline NOC & $59.50 \pm 8.18$ & $51.60 \pm 12.20$ & \\
NOWFI & $58.80 \pm 9.80$ & $64.70 \pm 10.55$ & $F(3,35)=17.378$ \\
OC & $42.60 \pm 8.74$ & $44.50 \pm 10.49$ & $\begin{array}{l}p<.001 \\
\eta_{\mathrm{p}}^{2}=.598\end{array}$ \\
OWFI & $40.10 \pm 5.17$ & $66.20 \pm 6.56$ & \\
\hline
\end{tabular}

Note. $\quad$ NOC $=$ Non Obese Control; NOWFI $=$ Non Obese $\mathrm{Wii}$ Fit Intervention; OC = Obese Control; OWFI = Obese Wii Fit Intervention. women. Irrespective of the similar effectiveness of both groups in participating in Wii Fit games in their static balance, it was obvious that the obese college women benefit more than non-obese ones, but regarding the motor competence, more progress was made in the obese college women than the non-obese college women. Much earlier, Staiano et al. $(2018,2017)$ have shown the advantage of using exergame interventions in overweight and obese communities.

The results showed an average increase of $12 \%$ in the static balance of the non-obese and $20 \%$ in the obese college women. In this regard, the increase of concentration and coordination between mind and body level might be resulted from participating in Wii Fit balance games such as traditional balance exercises so that these people could keep their bodies in a more balanced and correct state, and this awareness could improve the response speed of motor systems, nervous systems, and proprioceptive systems ( $\mathrm{Yu} \&$ Yang, 2012). The good point about video games is that they need a quick reaction to succeed in these games. Thus, these games are regarded as a reaction time exercises. Reaction time exercises essentially represent the cognitive-motor interaction, which is crucial for balance recovery (Blomkvist et al., 2017), and Wii Fit exergames may increase balance by reinforcing it.

A recent study found that real yoga training, which is part of virtual reality Wii Fit games too, has a significant positive effect on the static and dynamic balance of obese young women. It seems that the neuromuscular adaptation obtained after these exercises improves the balance (Jorrakate, Kongsuk, Pongduang, Sadsee, \& Chanthorn, 2015). Many previous studies have shown the positive effect of Wii Fit exercises on balance improvement of different communities. Recently, Yazgan et al. (2020) showed that a Wii Fit exercise can significantly improve balance in patients affected by multiple sclerosis. Siriphorn and Chamonchant (2015) suggested that a Wii Balance Board exercise program can be used to improve the balance and lower limb muscle strength of overweight young adults. In addition, a relatively short-term intervention (10 30-minute sessions during 5 weeks) on patients with Parkinson's disease, compared to previous interventions that reported the efficacy of at least 14 training sessions to improve their balance, showed the short-term intervention can have a significant positive effect on the balance (Negrini et al., 2017).

One of the important features of Wii Fit games, like many virtual reality games, is that each person observes his/her action in the model of another person on the screen and/or follows a movement pattern to do the right move. This feature activates the mechanism of neural activation and subsequent reorganization in 
the primary sensorimotor cortex and stimulates mirror neurons, which are effective in improving motor skills (Gatica-Rojas et al., 2017; Negrini et al., 2017).

Another important factor in the effectiveness of Wii Fit exercises may concern the feedback provided by the system because it has been shown that augmented feedbacks are useful for enhancing motor learning. These feedbacks can help correct the movement or increase motivation by giving information about the correctness and success of the exercise (Gioftsidou et al., 2013). These feedbacks are also provided by visual, vestibular and auditory systems, all of which are related to balance (Gatica-Rojas et al., 2017).

One of the benefits of the present research was the presence of the trainer in the training sessions. The previous studies have shown that the presence of the trainer increases the intrinsic motivation of participants during bouts of exergaming (Staiano et al., 2017). In addition, as Wii Fit training programs are performed in the framework of a game-based model, they are more appealing to the youth than a traditional physical activity, and this activity can increase one's motivation to participate in it and strive for doing so (Cho, Hwang, Hwang, \& Chung, 2016; de Vries, Faber, Jonkers, Van Dieen, \& Verschueren, 2018; Gao, Chen, Pasco, \& Pope, 2015).

In addition, the difficulty levels of the Wii Fit games can generally be adjusted, and they also have a variety of training levels with varying degree of difficulty, allowing the player to choose from a variety of exercises with the appropriate difficulty levels in order to provide a suitable challenge throughout the training period. As a result, that is necessary to help improve the balance or motor performance (de Vries et al., 2018; Willaert et al., 2020).

To date, most research has shown the effect of motor interventions on motor proficiency in obese children or clinical populations, but the current study seeks to investigate the obese and non-obese college women whose motor skills are still developing and hence deficits may be more malleable to refinement (Page, Barrington, Edwards, \& Barnett, 2017). Cliff et al. (2011) reported that physical activity regardless of diet can improve obese children's motor proficiency. Another study suggested that a community-based physical activity motor development program, called SHARK, could improve motor development in obese children (Cliff, Wilson, Okely, Mickle, \& Steele, 2007).

In particular, Bonney, Ferguson, Burgess, and SmitsEngelsman (2019) reported that both Wii Fit intervention and task-oriented functional training may improve aerobic fitness and motor coordination in female adolescents who are overweight and obese. In addition, a study showed that 6-week intervention on the Nintendo
Wii can improve the level of functional fitness in obese children. In this study, the coordination, reaction time, speed and agility increased by magnitudes of 28\%, 94\% and $37 \%$ respectively (Van Biljon, \& Longhurst, 2012). In contrast, Weigmann-Faßbender et al. (2019) also showed that the intervention of home-based exergaming (Wii Fit) cannot improve cardiorespiratory fitness, motor competence, and quality of life in pediatric renal transplant recipients and that it merely increases their daily physical activity. One of the reasons for the ineffectiveness of the Wii Fit intervention in this study was lack of regular exercise program by the intervention group so that only 5 of the 13 young patients could correctly and regularly perform the game protocol. However, in the present study, all training sessions were conducted by the direct supervision of the trainer, and the researcher made sure that the training protocol was correctly implemented.

Like any other study, this study was not devoid of limitations. It can be pointed out that one of the limitations of this study is the small sample size and failure to use laboratory tools (such as force plates) in order to more accurately measure the balance. As a result, the results obtained this way are less comparable with those of other studies in which different devices were used. In addition, in the future research studies, it is recommended to simultaneously investigate the changes of BMI or body composition as well as other physical, motor, or mental changes in overweight groups so as to specify whether the resulting changes to exergames are due to adjustment for body composition/BMI or, or as discussed in this study, they can directly create positive effects through the mentioned mechanisms or other types of mechanisms.

\section{Conclusions}

Wii Fit exergames can be used as a suitable alternative for physical activities of non-obese and especially obese college women, provided that they are unable to participate in sports programs, thereby it can help improve motor competence and static balance.

\section{Conflict of interest}

There were no conflicts of interest.

\section{References}

Błaszczyk, J. W., Cieślinska-Świder, J., Plewa, M., ZahorskaMarkiewicz, B., \& Markiewicz, A. (2009). Effects of 
excessive body weight on postural control. Journal of Biomechanics, 42, 1295-1300.

Blomkvist, A. W., Eika, F., Rahbek, M. T., Eikhof, K. D., Hansen, M. D., Søndergaard, M., ... Jørgensen, M. G. (2017). Reference data on reaction time and aging using the Nintendo Wii Balance Board: A cross-sectional study of 354 subjects from 20 to 99 years of age. PLOS ONE, 12, e0189598.

Bonney, E., Ferguson, G., Burgess, T., \& Smits-Engelsman, B. (2019). Benefits of activity-based interventions among female adolescents who are overweight and obese. Pediatric Physical Therapy, 31, 338-345.

Bruininks, R. H., \& Bruininks, B. D. (2005). Bruininks-Oseretsky test of motor proficiency: Examiner's manual. Second edition. Windsor, United Kingdom: NFER-Nelson.

Cattuzzo, M. T., dos Santos Henrique, R., Ré, A. H. N., de Oliveira, I. S., Melo, B. M., de Sousa Moura, M., ... Stodden, D. (2016). Motor competence and health related physical fitness in youth: A systematic review. Journal of Science and Medicine in Sport, 19, 123-129.

Cho, C., Hwang, W., Hwang, S., \& Chung, Y. (2016). Treadmill training with virtual reality improves gait, balance, and muscle strength in children with cerebral palsy. Tohoku Journal of Experimental Medicine, 238, 213-218.

Cliff, D. P., Okely, A. D., Morgan, P. J., Steele, J. R., Jones, R. A., Colyvas, K. I. M., \& Baur, L. A. (2011). Movement skills and physical activity in obese children: Randomized controlled trial. Medicine \& Science in Sports \& Exercise, 43, 90-100.

Cliff, D. P., Wilson, A., Okely, A. D., Mickle, K. J., \& Steele, J. R. (2007). Feasibility of SHARK: A physical activity skill-development program for overweight and obese children. Journal of Science and Medicine in Sport, 10, 263-267.

Cluskey, M., \& Grobe, D. (2009). College weight gain and behavior transitions: Male and female differences. Journal of the American Dietetic Association, 109, 325-329.

Crocker, R. (2012). Determining the reliability of the Wii Balance Board ${ }^{T M}$ as a postural stability assessment tool (Master's thesis). Available from ProQuest Dissertations and Theses. (UMI No. 1520318)

de Vries, A. W., Faber, G., Jonkers, I., Van Dieen, J. H., \& Verschueren, S. M. (2018). Virtual reality balance training for elderly: Similar skiing games elicit different challenges in balance training. Gait \& Posture, 59, 111-116.

D’Hondt, E., Deforche, B., Gentier, I., De Bourdeaudhuij, I., Vaeyens, R., Philippaerts, R., \& Lenoir, M. (2013). A longitudinal analysis of gross motor coordination in overweight and obese children versus normal-weight peers. International Journal of Obesity, 37, 61-67.

Field, A. (2010). Discovering statistics using SPSS (4th ed.). London, United Kingdom: SAGE.

Gao, Z., Chen, S., Pasco, D., \& Pope, Z. (2015). A metaanalysis of active video games on health outcomes among children and adolescents. Obesity Reviews, 16, 783-794.

Gatica-Rojas, V., Méndez-Rebolledo, G., Guzman-Muñoz, E., Soto-Poblete, A., Cartes-Velásquez, R., ElguetaCancino, E., \& Cofré Lizama, E. (2017). Does Nintendo Wii Balance Board improve standing balance? A randomized controlled trial in children with cerebral palsy. European Journal of Physical and Rehabilitation Medicine, 53, 535-544.
Gioftsidou, A., Vernadakis, N., Malliou, P., Batzios, S., Sofokleous, P., Antoniou, P., ... Godolias, G. (2013). Typical balance exercises or exergames for balance improvement? Journal of Back and Musculoskeletal Rehabilitation, 26, 299-305.

Hands, B., Licari, M., \& Piek, J. (2015). A review of five tests to identify motor coordination difficulties in young adults. Research in Developmental Disabilities, 41, 40-51.

Jacobs, K., Zhu, L., Dawes, M., Franco, J., Huggins, A., Igari, C., ... Umez-Eronini, A. (2011). Wii health: A preliminary study of the health and wellness benefits of Wii Fit on university students. British Journal of Occupational Therapy, 74, 262-268.

Jorrakate, C., Kongsuk, J., Pongduang, C., Sadsee, B., \& Chanthorn, P. (2015). Effect of yoga training on one leg standing and functional reach tests in obese individuals with poor postural control. Journal of Physical Therapy Science, 27, 59-62.

McMahon, E. M., Corcoran, P., O’Regan, G., Keeley, H., Cannon, M., Carli, V., ... Balazs, J. (2017). Physical activity in European adolescents and associations with anxiety, depression and well-being. European Child \& Adolescent Psychiatry, 26, 111-122.

Negrini, S., Bissolotti, L., Ferraris, A., Noro, F., Bishop, M. D., \& Villafañe, J. H. (2017). Nintendo Wii Fit for balance rehabilitation in patients with Parkinson's disease: A comparative study. Journal of Bodywork and Movement Therapies, 21, 117-123.

Ng, Y. L., Ma, F., Ho, F. K., Ip, P., \& Fu, K. W. (2019). Effectiveness of virtual and augmented reality-enhanced exercise on physical activity, psychological outcomes, and physical performance: A systematic review and meta-analysis of randomized controlled trials. Computers in Human Behavior, 99, 278-291.

Nunez-Gaunaurd, A., Moore, J. G., Roach, K. E., Miller, T. L., \& Kirk-Sanchez, N. J. (2013). Motor proficiency, strength, endurance, and physical activity among middle school children who are healthy, overweight, and obese. Pediatric Physical Therapy, 25, 130-138.

Page, Z. E., Barrington, S., Edwards, J., \& Barnett, L. M. (2017). Do active video games benefit the motor skill development of non-typically developing children and adolescents: A systematic review. Journal of Science and Medicine in Sport, 20, 1087-1100.

Park, D. S., \& Lee, G. (2014). Validity and reliability of balance assessment software using the Nintendo Wii balance board: Usability and validation. Journal of NeuroEngineering and Rehabilitation, 11, 99.

Silva, V., Campos, C., Sá, A., Cavadas, M., Pinto, J., Simões, P., ... Barbosa-Rocha, N. (2017). Wii-based exercise program to improve physical fitness, motor proficiency and functional mobility in adults with Down syndrome. Journal of Intellectual Disability Research, 61, 755-765.

Siriphorn, A., \& Chamonchant, D. (2015). Wii balance board exercise improves balance and lower limb muscle strength of overweight young adults. Journal of Physical Therapy Science, 27, 41-46.

Staiano, A. E., Beyl, R. A., Guan, W., Hendrick, C. A., Hsia, D. S., \& Newton, R. L. (2018). Home-based exergaming among children with overweight and obesity: A randomized clinical trial. Pediatric Obesity, 13, 724-733. 
Staiano, A. E., Marker, A. M., Beyl, R. A., Hsia, D. S., Katzmarzyk, P. T., \& Newton, R. L. (2017). A randomized controlled trial of dance exergaming for exercise training in overweight and obese adolescent girls. Pediatric Obesity, 12, 120-128.

Tarakci, D., Ersoz Huseyinsinoglu, B., Tarakci, E., \& Razak Ozdincler, A. (2016). Effects of Nintendo Wii-Fit ${ }^{\circledR}$ video games on balance in children with mild cerebral palsy. Pediatrics International, 58, 1042-1050.

Taymoori, P., Niknami, S. H., Berry, T., Ghofranipour, F., \& Kazemnejad, A. (2009). Application stage of change exercise behavior among Iranian adolescents. Eastern Mediterranean Health Journal, 15, 785-795.

Taymoori, P., Rhodes, R. E., \& Berry, T. R. (2010). Application of a social cognitive model in explaining physical activity in Iranian female adolescents. Health Education Research, 25, 257-267.

Tripette, J., Murakami, H., Ryan, K. R., Ohta, Y., \& Miyachi, M. (2017). The contribution of Nintendo Wii Fit series in the field of health: A systematic review and meta-analysis. PeerJ, 5, e3600.

Van Biljon, A., \& Longhurst, G. K. (2012). The influence of exergaming on the functional fitness in overweight and obese children: Physical activity, health and wellness.
African Journal for Physical Health Education, Recreation and Dance, 18, 984-991.

Weigmann-Faßbender, S., Pfeil, K., Betz, T., Sander, A., Weiß, K., Tönshoff, B., \& Friedmann-Bette, B. (2019). Physical fitness and health-related quality of life in pediatric renal transplant recipients: An interventional trial with active video gaming. Pediatric Transplantation, 24, e13630.

Willaert, J., De Vries, A. W., Tavernier, J., Van Dieen, J. H., Jonkers, I., \& Verschueren, S. (2020). Does a novel exergame challenge balance and activate muscles more than existing off-the-shelf exergames? Journal of NeuroEngineering and Rehabilitation, 17, 6.

Yazgan, Y. Z., Tarakci, E., Tarakci, D., Ozdincler, A. R., \& Kurtuncu, M. (2020). Comparison of the effects of two different exergaming systems on balance, functionality, fatigue, and quality of life in people with multiple sclerosis: A randomized controlled trial. Multiple Sclerosis and Related Disorders, 39, 101902.

Yu, D. H., \& Yang, H. X. (2012). The effect of Tai Chi intervention on balance in older males. Journal of Sport and Health Science, 1, 57-60.

Zech, A., Hübscher, M., Vogt, L., Banzer, W., Hänsel, F., \& Pfeifer, K. (2010). Balance training for neuromuscular control and performance enhancement: A systematic review. Journal of Athletic Training, 45, 392-403. 